Pacific

Journal of

Mathematics

GRADIENT ESTIMATES FOR SOLUTIONS OF THE HEAT EQUATION UNDER RICCI FLOW

SHIPING LIU

Volume $243 \quad$ No. 1

November 2009 


\title{
GRADIENT ESTIMATES FOR SOLUTIONS OF THE HEAT EQUATION UNDER RICCI FLOW
}

\author{
SHIPING LIU
}

\begin{abstract}
We establish first order gradient estimates for positive solutions of the heat equations on complete noncompact or closed Riemannian manifolds under Ricci flows. These estimates improve Guenther's results by weakening the curvature constraints. We also obtain a result for arbitrary solutions on closed manifolds under Ricci flows. As applications, we derive Harnacktype inequalities and second order gradient estimates for positive solutions of the heat equations under Ricci flow. The results in this paper can be considered as generalizing the estimates of $\mathrm{Li}-$ Yau and J. Y. Li to the Ricci flow setting.
\end{abstract}

\section{Introduction}

In this paper, we mainly generalize Li and Yau's [1986] and Li's [1991] gradient estimates to positive solutions of the heat equation under Ricci flow. The Ricci flow,

$$
\partial_{t} g_{i j}=-2 \mathrm{Ric}_{i j},
$$

was introduced by Hamilton [1982] to study the Poincaré conjecture on compact three manifolds with positive Ricci curvature. Since then, in the series [1995; 1997; 1999], Hamilton created a well-developed theory of Ricci flow as an approach to the Poincaré conjecture and the geometrization conjecture. In [2002; 2003], Perelman brought in new ideas and completed the so-called Hamilton program.

Gradient estimates for solutions of the heat equation are very powerful tools in analysis, as shown for example in [Li 1991; Li and Yau 1986]. Perelman [2002] actually showed a gradient estimate for the fundamental solution of the conjugate heat equation,

$$
\Delta u-R u+\partial_{t} u=0,
$$

under Ricci flow on a closed Riemannian manifold $M$, where $R$ is the scalar curvature. Namely, let $u$ be the fundamental solution of the equation above in $M \times[0, T)$,

MSC2000: primary 58J35, 53C44; secondary 35K55, 53C21.

Keywords: gradient estimate, Ricci flow, heat equation, Harnack inequality. 
and let $f$ be the function such that $u=(4 \pi \tau)^{-n / 2} e^{-f}$ with $\tau=T-t$. Then

$$
\left(\tau\left(2 \Delta f-|\nabla f|^{2}+R\right)+f-n\right) u \leq 0 \quad \text { in } M \times[0, T) .
$$

Equivalently,

$$
\frac{|\nabla u|^{2}}{u^{2}}-\frac{u_{\tau}}{u}-R \leq \frac{n}{\tau}+\frac{\ln u}{\tau}+\frac{n}{2} \frac{\ln (4 \pi \tau)}{\tau} .
$$

This estimate is important in the proof of Perelman. Recently, Kuang and Zhang [2008] established a gradient estimate that works for all positive solutions of the conjugate heat equation under Ricci flow on a closed manifold. (Here and throughout we say that a Riemannian manifold is closed if it is compact without boundary.) As an immediate consequence, they get a Harnack-type inequality. By supposing a lower bound on the Ricci curvature, Zhang [2006] established local gradient estimates for positive solutions of the heat equation under the backward Ricci flow $\partial_{t} g_{i j}=2 \mathrm{Ric}_{i j}$ on a closed Riemannian manifold. Under stronger curvature constraints, Guenther [2002] had already established gradient estimates for positive solutions of the heat equation under Ricci flow on a closed manifold. Using this result, she derived a Harnack-type inequality and found a lower bound for the heat kernel under Ricci flow. Here heat kernel means the fundamental solution of the heat equation under Ricci flow, whose existence and basic properties Guenther also proved. We weaken her curvature constraints in Section 2 using the method of $\mathrm{Li}$ and Yau [1986]. We also get corresponding estimates for complete noncompact manifolds under Ricci flows. All of these results are generalizations of $\mathrm{Li}$ and Yau's gradient estimates.

Interesting in their own right, higher order gradient estimates for heat kernels on complete noncompact Riemannian manifolds under Ricci flows are also closely related with the boundedness of the Riesz transform and the Sobolev inequality. Zhang [2007] found that the noncollapsing result, which is critical in Perelman's proof of the Poincaré conjecture, follows immediately from the Sobolev inequality under Ricci flow. Li [1994] used the boundedness of the Riesz transform to prove the Sobolev inequality on Riemannian manifold with some constraints, so it is natural to try to prove with a similar method the Sobolev inequality under Ricci flow. In that method, an important step, completed [Li 1991], is to prove an estimate for $\nabla_{X} \nabla_{Y} H(x, y, t)$, where $H(x, y, t)$ is the heat kernel and $\nabla_{X}$ and $\nabla_{Y}$ are the gradient operator in the variables $x$ and $y$. However, difficulties arise in using this method to get the generalization of this estimate under Ricci flow, since in this case the heat kernel has properties different from what it had in the fixed metric case. As a consequence of the first order results in Section 2, in Section 3 we get the generalization of Li's [1991] second order gradient estimate for the positive solution $u(x, t)$ of the heat equation under Ricci flow. Let $M$ be a complete noncompact Riemannian manifold with initial metric $g(0)$. Assume that $g(t)$ evolves 
by Equation (1-1) and its first order covariant derivatives are bounded by $k_{1}$ and $k_{2}$. Then we have (the notation is defined in later sections)

$$
\frac{\left|\nabla^{2} u(x, t)\right|}{u(x, t)}+\alpha \frac{|\nabla u(x, t)|^{2}}{u^{2}(x, t)}-5 \alpha \frac{u_{t}(x, t)}{u(x, t)} \leq C\left(k_{1}+k_{2}^{2 / 3}+1 / t\right) .
$$

In fact, the estimate for $\nabla_{X} \nabla_{Y} H(x, y, t)$ and the second order gradient estimate for $u(x, t)$ are proved similarly in [Li 1991]. The main difference is that the latter doesn't depend on the special properties of the heat kernel.

For closed Riemannian manifolds under Ricci flows, we get a gradient estimate for arbitrary solutions of the heat equation at the end of Section 2.

We will use the following notations: We denote by $\nabla$ and $\Delta$ the gradient and Laplacian-Beltrami operator under the metric $g(t)$; by $C$ a positive constant that may change from line to line; by $d(x, y, t)$ the geodesic distance between $x, y \in M$ under $g(t)$; and by $\psi(r)$ a $C^{2}$ function on $[0,+\infty)$, such that

$$
\psi(r)= \begin{cases}1 & \text { if } r \in[0,1] \\ 0 & \text { if } r \in[2,+\infty)\end{cases}
$$

and

$$
0 \leq \psi(r) \leq 1, \quad \psi^{\prime}(r) \leq 0, \quad \psi^{\prime \prime}(r) \geq-C, \quad \frac{\left|\psi^{\prime}(r)\right|^{2}}{\psi(r)} \leq C,
$$

where $C$ is an absolute constant. When we say that $u(x, t)$ is a solution to the heat equation, we mean $u$ is a solution that is $C^{2}$ in $x$ and $C^{1}$ in $t$.

\section{The first order gradient estimates}

In this section, we prove the first order gradient estimates. We will denote

$$
f_{t}(x, t)=\partial_{t} f(x, t)=\frac{\partial f(x, t)}{\partial t}
$$

for a function $f$ on $M \times[0, T]$, where $T$ is a positive constant. We give a local version gradient estimate first.

Theorem 1. Let $g(t)$ be a solution to the Ricci flow on a Riemannian manifold $M^{n}$ with $n \geq 2$ for $t$ in some time interval $[0, T]$, and suppose $-K_{0} \leq \mathrm{Ric} \leq K_{1}$ for some positive constants $K_{0}$ and $K_{1}$ and all $t \in[0, T]$. Let $M$ be complete under the initial metric $g(0)$. Given $x_{0} \in M$ and $R>0$, let $u$ be a positive solution to the equation $\left(\Delta-\partial_{t}\right) u(x, t)=0$ in the cube $Q_{2 R, T}:=\left\{(x, t) \mid d\left(x, x_{0}, t\right) \leq 2 R, 0 \leq t \leq T\right\}$. Then for $(x, t) \in Q_{R, T}$, we have

$$
\frac{|\nabla u(x, t)|^{2}}{u^{2}(x, t)}-\alpha \frac{u_{t}(x, t)}{u(x, t)} \leq C\left(K_{1}+K_{0}+\frac{1}{t}+\frac{1}{R^{2}}\right)
$$

for any $\alpha>1$, where $C$ depends on $n$ and $\alpha$ only. 
More explicitly, we have

$$
\begin{aligned}
\frac{|\nabla u(x, t)|^{2}}{u^{2}(x, t)}-\alpha \frac{u_{t}(x, t)}{u(x, t)} \leq \frac{n \alpha^{2}}{t} & +\frac{C \alpha^{2}}{R^{2}}\left(R \sqrt{K_{0}}+\frac{\alpha^{2}}{\alpha-1}\right) \\
& +\frac{n \alpha^{3}}{\alpha-1} K_{0}+n^{3 / 2} \alpha^{2}\left(K_{0}+K_{1}\right)+C \alpha^{2} K_{1},
\end{aligned}
$$

for any $\alpha>1$, where $C$ depends on $n$ only.

As in the proof in [Li and Yau 1986], let $f=\log u$, and let

$$
F=t\left(\frac{|\nabla u(x, t)|^{2}}{u^{2}(x, t)}-\alpha \frac{u_{t}(x, t)}{u(x, t)}\right)=t\left(|\nabla f|^{2}-\alpha f_{t}\right) .
$$

Lemma 1. Suppose $(M, g(t))$ satisfies the hypotheses of Theorem 1. We have

$$
\begin{aligned}
\left(\Delta-\partial_{t}\right) F \geq-2 \nabla f \cdot \nabla F+\frac{t}{n}\left(|\nabla f|^{2}\right. & \left.-f_{t}\right)^{2}-\left(|\nabla f|^{2}-\alpha f_{t}\right) \\
& -2 \alpha K_{0} t|\nabla f|^{2}-t \alpha^{2} n^{2}\left(K_{0}+K_{1}\right)^{2} .
\end{aligned}
$$

Proof. For a given time $t$, choose $\left\{x_{1}, x_{2}, \ldots, x_{n}\right\}$ to be a normal coordinate system at a fixed point. The subscripts $i$ and $j$ will denote covariant derivatives in the $x_{i}$ and $x_{j}$ directions. We will compute at the fixed point.

By a direct computation, we obtain

$$
\Delta F=t\left(2 \sum_{i, j} f_{i j}^{2}+2 \sum_{i, j} f_{i} f_{j j i}+2 \sum_{i, j} \operatorname{Ric}_{i j} f_{i} f_{j}-\alpha(\Delta f)_{t}+2 \alpha \sum_{i, j} \operatorname{Ric}_{i j} f_{i j}\right),
$$

where we have used the Ricci identity and the formula

$$
\Delta\left(f_{t}\right)=(\Delta f)_{t}-2\langle\operatorname{Ric}, \operatorname{Hess}(f)\rangle .
$$

On the other hand, we have

$$
F_{t}=\left(|\nabla f|^{2}-\alpha f_{t}\right)+t\left(2 \sum_{i, j} \operatorname{Ric}_{i j} f_{i} f_{j}+2 \sum_{i} f_{t i} f_{i}-\alpha f_{t t}\right) .
$$

Then noting that $\left(\Delta-\partial_{t}\right) f=-|\nabla f|^{2}$, we arrive at

$$
\begin{aligned}
\left(\Delta-\partial_{t}\right) F=-2 \nabla f \cdot \nabla F+2 t\left(\sum f_{i j}^{2}\right. & \left.+\alpha \sum \operatorname{Ric}_{i j} f_{i j}\right) \\
& +2 \alpha t \operatorname{Ric}(\nabla f, \nabla f)-\left(|\nabla f|^{2}-\alpha f_{t}\right) .
\end{aligned}
$$

Because $\left(\operatorname{Ric}_{i j}\right)_{n \times n}$ is a real symmetric matrix, we obtain

$$
-K_{0}-K_{1} \leq \operatorname{Ric}_{i j} \leq K_{1}+K_{0}
$$

from $-K_{0} \leq$ Ric $\leq K_{1}$. Applying those bounds and Young's inequality in the form

$$
\left|\operatorname{Ric}_{i j}\right|\left|f_{i j}\right| \leq \frac{\alpha}{2} \operatorname{Ric}_{i j}^{2}+\frac{1}{2 \alpha} f_{i j}^{2},
$$


we conclude

$$
\begin{aligned}
\left(\Delta-\partial_{t}\right) F \geq-2 \nabla f \cdot \nabla F+t \sum f_{i j}^{2}-t \alpha^{2} & n^{2}\left(K_{0}+K_{1}\right)^{2} \\
& -2 \alpha K_{0} t|\nabla f|^{2}-\left(|\nabla f|^{2}-\alpha f_{t}\right) .
\end{aligned}
$$

The lemma is completed with the help of the inequality

$$
\sum_{i, j} f_{i j}^{2} \geq \sum_{i} f_{i i}^{2} \geq \frac{1}{n}(\Delta f)^{2}=\frac{1}{n}\left(|\nabla f|^{2}-f_{t}\right)^{2} .
$$

Proof of Theorem 1. Bounded Ricci curvature implies that $g(t)$ is uniformly equivalent to the initial metric $g(0)$ (see [Chow et al. 2006, Corollary 6.11]), that is,

$$
e^{-2 K_{1} T} g(0) \leq g(t) \leq e^{2 K_{0} T} g(0) .
$$

By definition, we know that $(M, g(t))$ is also complete for $t \in[0, T]$.

Let

$$
\varphi(x, t)=\varphi\left(d\left(x, x_{0}, t\right)\right)=\psi\left(\frac{d\left(x, x_{0}, t\right)}{R}\right)=\psi\left(\frac{\rho(x, t)}{R}\right),
$$

where $\rho(x, t)=d\left(x, x_{0}, t\right)$. For the purpose of applying the maximum principle, the argument of [Calabi 1958] allows us to assume that the function $\varphi(x, t)$, with support in $Q_{2 R, T}$, is $C^{2}$ at the maximum point.

For any $0<T_{1} \leq T$, let $\left(x_{1}, t_{1}\right)$ be the point in $Q_{2 R, T_{1}}$ at which $\varphi F$ achieves its maximum value. We can assume that this value is positive, because otherwise the proof is trivial. Then at the point $\left(x_{1}, t_{1}\right)$, we have

$$
\nabla(\varphi F)=F \nabla \varphi+\varphi \nabla F=0, \quad \Delta(\varphi F) \leq 0, \quad \partial_{t}(\varphi F) \geq 0 .
$$

Therefore,

$$
\begin{aligned}
0 & \geq\left(\Delta-\partial_{t}\right)(\varphi F) \\
& =(\Delta \varphi) F+\varphi\left(\Delta-\partial_{t}\right) F-\varphi_{t} F+2 \nabla \varphi \nabla F .
\end{aligned}
$$

Using the Laplacian comparison theorem, we have

$$
\Delta \varphi \geq-\frac{C}{R^{2}}-\frac{C}{R} \sqrt{K_{0}} .
$$

By the evolution formula of the geodesic length under Ricci flow (see [Chow and Knopf 2004]), we calculate

$$
\begin{aligned}
-F \varphi_{t} & =-F \psi^{\prime}\left(\frac{\rho}{R}\right) \frac{1}{R} \frac{d \rho}{d t}=F \psi^{\prime}\left(\frac{\rho}{R}\right) \frac{1}{R} \int_{\gamma_{t_{1}}} \operatorname{Ric}(S, S) d s \\
& \geq F \psi^{\prime}\left(\frac{\rho}{R}\right) \frac{1}{R} K_{1} \rho F \psi^{\prime}\left(\frac{\rho}{R}\right) K_{1} \geq-F \sqrt{C} K_{1},
\end{aligned}
$$


where $\gamma_{t_{1}}$ is the geodesic connecting $x$ and $x_{0}$ under the metric $g\left(t_{1}\right), \quad S$ is the unit tangent vector to $\gamma_{t_{1}}$, and $d s$ is the element of arc length. Substituting the two inequalities above into (2-9) and using (2-8), we obtain

$$
0 \geq\left(-\frac{C}{R^{2}}-\frac{C}{R} \sqrt{K_{0}}\right) F-\sqrt{C} K_{1} F+\varphi\left(\Delta-\partial_{t}\right) F .
$$

Applying Lemma 1 to this inequality yields

$$
\begin{array}{r}
0 \geq\left(-\frac{C}{R^{2}}-\frac{C}{R} \sqrt{K_{0}}\right) F-\sqrt{C} K_{1} F-2 \frac{\sqrt{C}}{R} \sqrt{\varphi}|\nabla f| F+\frac{t_{1}}{n} \varphi\left(|\nabla f|^{2}-f_{t}\right)^{2} \\
-\varphi\left(|\nabla f|^{2}-\alpha f_{t}\right)-2 \alpha K_{0} t_{1} \varphi|\nabla f|^{2}-t_{1} \alpha^{2} n^{2} \varphi\left(K_{1}+K_{0}\right)^{2}
\end{array}
$$

The following computation is almost the same as one in [Li and Yau 1986]. Multiplying through by $\varphi t_{1}$ and setting $y=\varphi|\nabla f|^{2}$ and $z=\varphi f_{t}$, Equation (2-11) becomes

$$
\begin{aligned}
0 \geq t_{1}\left(-\frac{C}{R^{2}}-\right. & \left.\frac{C}{R} \sqrt{K_{0}}\right)(\varphi F)-\sqrt{C} K_{1} t_{1}(\varphi F)-2 \frac{\sqrt{C}}{R} t_{1}^{2} y^{1 / 2}(y-\alpha z) \\
& +\frac{t_{1}^{2}}{n}(y-z)^{2}-2 \alpha K_{0} t_{1}^{2} y-\varphi^{2} F-t_{1}^{2} \alpha^{2} n^{2} \varphi^{2}\left(K_{0}+K_{1}\right)^{2}
\end{aligned}
$$

Using the inequality $a x^{2}-b x \geq-b^{2} /(4 a)$ for $a, b>0$, one obtains

$$
\begin{aligned}
& \frac{t_{1}^{2}}{n}(y-z)^{2}-2 \frac{\sqrt{C}}{R} t_{1}^{2} y^{1 / 2}(y-\alpha z)-2 \alpha K_{0} t_{1}^{2} y \\
& \quad=\frac{t_{1}^{2}}{n}\left(\frac{1}{\alpha^{2}}(y-\alpha z)^{2}+\left(\frac{\alpha-1}{\alpha}\right)^{2} y^{2}-2 \alpha n K_{0} y+\left(2 \frac{\alpha-1}{\alpha^{2}} y-\frac{2 n \sqrt{C}}{R} y^{1 / 2}\right)(y-\alpha z)\right) \\
& \quad \geq \frac{t_{1}^{2}}{n}\left(\frac{1}{\alpha^{2}}(y-\alpha z)^{2}-\frac{\alpha^{4} n^{2} K_{0}^{2}}{(\alpha-1)^{2}}-\frac{\alpha^{2} n^{2} C}{2(\alpha-1) R^{2}}(y-\alpha z)\right) .
\end{aligned}
$$

Hence (2-12) becomes

$$
\begin{aligned}
\frac{1}{n \alpha^{2}}(\varphi F)^{2}-(\varphi F)\left(1+\frac{C}{R^{2}} t_{1}\right. & \left.+\frac{C}{R} \sqrt{K_{0}} t_{1}+\frac{C n \alpha^{2} t_{1}}{2(\alpha-1) R^{2}}+\sqrt{C} K_{1} t_{1}\right) \\
- & \left(\frac{n K_{0}^{2} \alpha^{4} t_{1}^{2}}{(\alpha-1)^{2}}+t_{1}^{2} \alpha^{2} n^{2} \varphi^{2}\left(K_{1}+K_{0}\right)^{2}\right) \leq 0
\end{aligned}
$$

We apply the quadratic formula and then arrive at

$$
\begin{aligned}
\varphi F\left(x_{1}, t_{1}\right) \leq n \alpha^{2}+\frac{C n \alpha^{2}}{R^{2}}\left(R \sqrt{K_{0}}+\right. & \left.\frac{\alpha^{2}}{\alpha-1}\right) t_{1}+\sqrt{C} n \alpha^{2} K_{1} t_{1} \\
& +\frac{n \alpha^{3} t_{1}}{\alpha-1} K_{0}+t_{1} \varphi n^{3 / 2}\left(K_{0}+K_{1}\right) \alpha^{2} .
\end{aligned}
$$

This estimate for $\varphi F$ is also correct on $\left\{\left(x, T_{1}\right) \mid d\left(x, x_{0}, T_{1}\right) \leq 2 R\right\}$ since $t_{1} \leq T_{1}$. Since $T_{1}$ is arbitrary in $0<T_{1} \leq T$, we have completed the proof of Theorem 1 . 
The local result above implies a global one.

Corollary 1. Let $(M, g(0))$ be a complete noncompact Riemannian manifold without boundary, and suppose $g(t)$ evolves by Ricci flow in such a way that $-K_{0} \leq$ Ric $\leq K_{1}$ for $t \in[0, T]$. Let $u$ be a positive solution to the equation $\left(\Delta-\partial_{t}\right) u(x, t)=$ 0 . Then for $(x, t) \in M \times(0, T]$, we have

$$
\frac{|\nabla u(x, t)|^{2}}{u^{2}(x, t)}-\alpha \frac{u_{t}(x, t)}{u(x, t)} \leq \frac{n \alpha^{2}}{t}+C\left(K_{1}+K_{0}\right),
$$

for any $\alpha>1$, where $C$ depends on $n$ and $\alpha$ only.

Proof. By the uniform equivalence of $g(t)$, we know that $(M, g(t))$ is complete noncompact for $t \in[0, T]$. Then let $R \rightarrow+\infty$ in (2-2).

Remark 1. When $(M, g(0))$ is a complete noncompact Riemannian manifold, Shi [1989] gives a sufficient condition for the short time existence of the Ricci flow: It suffices that the curvature tensor $\left\{R_{i j k l}\right\}$ of $g(0)$ satisfies

$$
\left|R_{i j k l}\right|^{2} \leq \kappa \quad \text { on } \mathrm{M},
$$

where $0<\kappa<+\infty$ is a constant.

Using Lemma 1, we can also derive a similar gradient estimate on a closed Riemannian manifold.

Theorem 2. Let $(M, g(t))$ be a closed Riemannian manifold, where $g(t)$ evolves by Ricci flow in such a way that $-K_{0} \leq \mathrm{Ric} \leq K_{1}$ for $t \in[0, T]$. If $u$ is a positive solution to the equation $\left(\Delta-\partial_{t}\right) u(x, t)=0$, then for $(x, t) \in M \times(0, T]$, we have

$$
\frac{|\nabla u(x, t)|^{2}}{u^{2}(x, t)}-\alpha \frac{u_{t}(x, t)}{u(x, t)} \leq \frac{n \alpha^{2}}{t}+\frac{n \alpha^{3} K_{0}}{\alpha-1}+n^{3 / 2} \alpha^{2}\left(K_{0}+K_{1}\right),
$$

for any $\alpha>1$, where $C$ depends on $n$ and $\alpha$ only.

Proof. Let notations $F$ and $f$ be as above. Set

$$
\bar{F}(x, t)=F(x, t)-\frac{n \alpha^{3} K_{0}}{\alpha-1} t-n^{3 / 2} \alpha^{2}\left(K_{0}+K_{1}\right) t .
$$

If $\bar{F}(x, t) \leq n \alpha^{2}$ for any $(x, t) \in M \times(0, T]$, then the theorem is proved.

If (2-16) doesn't hold, then at the maximal point $\left(x_{0}, t_{0}\right)$ of $\bar{F}(x, t)$, we have $\bar{F}\left(x_{0}, t_{0}\right)>n \alpha^{2}$. Noting $\bar{F}(x, 0)=0$, we know here $t_{0}>0$. Then applying the maximal principle, we have at the point $\left(x_{0}, t_{0}\right)$ that

$$
\nabla \bar{F}\left(x_{0}, t_{0}\right)=0, \quad \Delta \bar{F}\left(x_{0}, t_{0}\right) \leq 0, \quad \frac{\partial}{\partial t} \bar{F}\left(x_{0}, t_{0}\right) \geq 0 .
$$

Therefore we obtain

$$
0 \geq\left(\Delta-\partial_{t}\right) \bar{F} \geq\left(\Delta-\partial_{t}\right) F .
$$


Using Lemma 1 and the trick in calculating (2-11), we get

$$
\begin{aligned}
0 \geq \frac{t_{0}}{n \alpha^{2}}\left(\frac{F}{t_{0}}\right)^{2}-\left(\frac{F}{t_{0}}\right)-\frac{n \alpha^{4} K_{0}^{2}}{(\alpha-1)^{2}} t_{0}-t_{0} \alpha^{2} n^{2}\left(K_{0}+\right. & \left.K_{1}\right)^{2} \\
& +\frac{2 t_{0}}{n} \frac{\alpha-1}{\alpha^{2}}|\nabla f|^{2} \frac{F}{t_{0}} .
\end{aligned}
$$

Since

$$
\frac{F}{t_{0}}=\frac{\bar{F}}{t_{0}}+\frac{n \alpha^{3} K_{0}}{\alpha-1}+n^{3 / 2} \alpha^{2}\left(K_{0}+K_{1}\right)>0,
$$

we get the inequality

$$
\frac{t_{0}}{n \alpha^{2}}\left(\frac{F}{t_{0}}\right)^{2}-\left(\frac{F}{t_{0}}\right)-\frac{n \alpha^{4} K_{0}^{2}}{(\alpha-1)^{2}} t_{0}-t_{0} \alpha^{2} n^{2}\left(K_{0}+K_{1}\right)^{2} \leq 0 .
$$

Again the quadratic formula gives

$$
\frac{F}{t_{0}} \leq \frac{n \alpha^{2}}{t_{0}}+\frac{n \alpha^{3} K_{0}}{\alpha-1}+n^{3 / 2} \alpha^{2}\left(K_{0}+K_{1}\right)
$$

This implies $\bar{F}\left(x_{0}, t_{0}\right) \leq n \alpha^{2}$, a contradiction. So (2-16) holds.

Remark 2. In Corollary 1 and Theorem 2 , if $K_{0}=0$, we can let $\alpha \rightarrow 1$.

In fact, the Theorem 2 improves the gradient inequality in [Guenther 2002], which requires the boundedness of the gradient of scalar curvature in addition to the boundedness of the Ricci curvature. Beginning with this result, we can do things similar to what was done in [Guenther 2002], such as deriving Harnacktype inequalities.

Corollary 2. Let $(M, g(0))$ be a complete noncompact Riemannian manifold without boundary or a closed Riemannian manifold, and suppose $g(t)$ evolves by Ricci flow for $t \in[0, T]$ in such a way that $-K_{0} \leq \mathrm{Ric} \leq K_{1}$. If $u$ is a positive solution to the equation $\left(\Delta-\partial_{t}\right) u(x, t)=0$, then for any points $\left(x, t_{1}\right),\left(y, t_{2}\right) \in M \times(0, T]$ such that $t_{1}<t_{2}$, we have

$$
u\left(x, t_{1}\right) \leq u\left(y, t_{2}\right)\left(\frac{t_{2}}{t_{1}}\right)^{2 n \varepsilon} \exp \left(\int_{0}^{1} \frac{\varepsilon\left|\gamma^{\prime}(s)\right|_{\sigma}^{2}}{2\left(t_{2}-t_{1}\right)} d s+C \frac{t_{2}-t_{1}}{2 \varepsilon}\left(K_{1}+K_{0}\right)\right)
$$

for any $\varepsilon>1 / 2$, where $C$ depends on $n$ and $\varepsilon$ only, $\gamma(s)$ is a smooth curve connecting $x$ and $y$ with $\gamma(1)=x$ and $\gamma(0)=y$, and $\left|\gamma^{\prime}(s)\right|_{\sigma}$ is the length of the vector $\gamma^{\prime}(s)$ at time $\sigma=(1-s) t_{2}+s t_{1}$.

Proof. First note that the gradient estimates in Corollary 1 and Theorem 2 can both be written as

$$
\frac{|\nabla u(x, t)|^{2}}{u^{2}(x, t)}-\alpha \frac{u_{t}(x, t)}{u(x, t)} \leq \frac{n \alpha^{2}}{t}+C_{n, \alpha}\left(K_{1}+K_{0}\right)
$$


Define $l(s)=\ln u\left(\gamma(s),(1-s) t_{2}+s t_{1}\right)$. It is easy to see that $l(0)=\ln u\left(y, t_{2}\right)$ and $l(1)=\ln u\left(x, t_{1}\right)$. Direct calculation gives

$$
\begin{aligned}
\frac{\partial l(s)}{\partial s} & =\left(t_{2}-t_{1}\right)\left(\frac{\nabla u}{u} \frac{\gamma^{\prime}(s)}{t_{2}-t_{1}}-\frac{u_{t}}{u}\right) \\
& \leq \frac{\varepsilon\left|\gamma^{\prime}(s)\right|_{\sigma}^{2}}{2\left(t_{2}-t_{1}\right)}+\frac{t_{2}-t_{1}}{2 \varepsilon}\left(C\left(K_{1}+K_{0}\right)+\frac{4 \varepsilon^{2} n}{\sigma(s)}\right) .
\end{aligned}
$$

Integrating this inequality over $\gamma(s)$, we have

$$
\begin{aligned}
\ln \frac{u\left(x, t_{1}\right)}{u\left(y, t_{2}\right)} & =\int_{0}^{1} \frac{\partial l(s)}{\partial s} d s \\
& \leq \int_{0}^{1} \frac{\varepsilon\left|\gamma^{\prime}(s)\right|_{\sigma}^{2}}{2\left(t_{2}-t_{1}\right)} d s+C \frac{t_{2}-t_{1}}{2 \varepsilon}\left(K_{1}+K_{0}\right)+2 \varepsilon n \ln \left(t_{2} / t_{1}\right) .
\end{aligned}
$$

We can also get a gradient estimate for an arbitrary solution of the heat equation under Ricci flow on a closed manifold without any curvature conditions. The auxiliary function $F$ we take in the following proof is inspired by Hamilton's [1995] proof of Shi's [1989] derivative estimates.

Theorem 3. Let $(M, g(t))$ be a closed Riemannian manifold, where $g(t)$ solves the Ricci flow for $t \in[0, T]$. If $u$ solves $\Delta u-\partial_{t} u=0$, then

$$
|\nabla u(x, t)|^{2} \leq \frac{1}{2 t}\left(\max _{x \in M} u^{2}(x, 0)-u^{2}(x, t)\right) \quad \text { for }(x, t) \in M \times[0, T] .
$$

Proof. Since $\partial_{t} u=\Delta u$, we have

$$
\partial_{t}\left(|\nabla u|^{2}\right)=2 \operatorname{Ric}(\nabla u, \nabla u)+2\langle\nabla u, \nabla(\Delta u)\rangle .
$$

Using Bochner's formula, this becomes

$$
\partial_{t}\left(|\nabla u|^{2}\right)=\Delta\left(|\nabla u|^{2}\right)-2\left|\nabla^{2} u\right|^{2} .
$$

On the other hand,

$$
\partial_{t}\left(u^{2}\right)=\Delta\left(u^{2}\right)-2|\nabla u|^{2} .
$$

Let $F=t|\nabla u|^{2}+A u^{2}$, where $A$ is a constant to be fixed. Then combining (2-25) and $(2-26)$ gives

$$
\begin{aligned}
\partial_{t} F & =|\nabla u|^{2}+t\left(\Delta\left(|\nabla u|^{2}\right)-2\left|\nabla^{2} u\right|^{2}\right)+A\left(\Delta\left(u^{2}\right)-2|\nabla u|^{2}\right) \\
& \leq \Delta F+(1-2 A)|\nabla u|^{2} .
\end{aligned}
$$

Setting $A=1 / 2$ and applying maximum principle, we conclude

$$
F(x, t) \leq \max _{x \in M} F(x, 0)=\frac{1}{2} \max _{x \in M} u^{2}(x, 0) .
$$

This inequality implies the theorem. 
Remark 3. For a positive solution of the heat equation $u$ on closed manifolds under Ricci flow, Zhang [2006] gives a stronger estimate,

$$
\frac{|\nabla u|}{u} \leq \sqrt{\frac{1}{t}} \sqrt{\ln \frac{M}{u}},
$$

where $M=\max _{x \in M} u(x, 0)$. Similar to the fact that the interpolation inequality follows from this estimate in [Zhang 2006], here we get for any $x, y \in M$ and $0<t \leq T$ that

$$
u(x, t) \leq u(y, t)+\sqrt{\frac{C}{2}} \frac{d(x, y, t)}{\sqrt{t}},
$$

where $C=\max _{x \in M} u^{2}(x, 0)$.

\section{The second order gradient estimates}

Using Corollary 1, we can generalize to the Ricci flow setting the second order gradient estimate for the positive solution of the heat equation in [Li 1991].

Theorem 4. Let $g(t)$ be a solution to the Ricci flow on a Riemannian manifold $M^{n}$ with $n \geq 2$ for $t$ in some time interval $[0, T]$. Assume that $(M, g(0))$ is a complete noncompact manifold without boundary. Suppose the curvature tensor and its first order covariant derivatives are bounded throughout by $k_{1}$ and $k_{2}$, respectively. Let $u$ be a positive solution to $\left(\Delta-\partial_{t}\right) u(x, t)=0$. Then for $(x, t) \in M \times(0, T]$,

$$
\frac{\left|\nabla^{2} u(x, t)\right|}{u(x, t)}+\alpha \frac{|\nabla u(x, t)|^{2}}{u^{2}(x, t)}-5 \alpha \frac{u_{t}(x, t)}{u(x, t)} \leq C\left(k_{1}+k_{2}^{2 / 3}+\frac{1}{t}\right),
$$

for any $\alpha>1$, where $C$ depends on $n$ and $\alpha$ only.

To prove the theorem, we set

$$
F(x, y, t)=t F_{1}=t\left(\frac{\left|\nabla^{2} u(x, t)\right|}{u(x, t)}+\alpha \frac{|\nabla u(x, t)|^{2}}{u^{2}(x, t)}-\beta \frac{u_{t}(x, t)}{u(x, t)}\right),
$$

where $\beta$ is a constant to be fixed.

Lemma 2. Suppose $(M, g(t))$ satisfies the hypotheses of Theorem 4. Then for sufficiently small $\delta>0$ and $\gamma-1>0$ and some $\varepsilon>0$, we have

$$
\begin{aligned}
\left(\Delta-\partial_{t}\right) F \geq-2 \nabla F \cdot & \nabla \log u+\frac{\delta \alpha}{t} F^{2}+2 \delta \alpha \beta F \frac{u_{t}}{u}-2 \delta \alpha^{2} F \frac{|\nabla u|^{2}}{u^{2}} \\
-C k_{1} F-\frac{C k_{1}^{2}}{4(\gamma-1)^{2}} t-C k_{2}^{4 / 3} t-4 \beta \varepsilon n^{2} k_{1}^{2} t-F / t & -2 C t\left(4 \delta \alpha^{3}+\frac{\delta}{2 \alpha(1-\delta)^{2}}\right)\left(\frac{1}{t}+k_{1}\right)^{2},
\end{aligned}
$$


where $\beta=5 \alpha$ and $C$ depends on $n$ and $\alpha$.

Proof. As in the proof of Lemma 1, choose $\left\{x_{1}, x_{2}, \ldots, x_{n}\right\}$ to be a normal coordinate system at a fixed point. Subscripts $i, j$ and $k$ will denote covariant derivatives in the $x_{i}, x_{j}$ and $x_{k}$ directions.

We will calculate the evolution equation for $F_{1}$. The computation is a little long, so we divide it into three parts.

Part 1. We calculate the equation for $\left|\nabla^{2} u\right| / u$.

It follows from [Li 1991] that

$$
\begin{array}{r}
\Delta\left(\frac{\left|\nabla^{2} u\right|}{u}\right)=\frac{\sum u_{i j k}^{2}+\sum u_{i j} u_{i j k k}}{u\left|\nabla^{2} u\right|}-2 \frac{\sum u_{i j} u_{i j k} u_{k}}{u^{2}\left|\nabla^{2} u\right|}-\frac{\sum_{k}\left(\sum_{i j} u_{i j} u_{i j k}\right)^{2}}{u\left|\nabla^{2} u\right|^{3}} \\
-\frac{\left|\nabla^{2} u\right| \Delta u}{u^{2}}+2 \frac{\left|\nabla^{2} u\right||\nabla u|^{2}}{u^{3}},
\end{array}
$$

$$
\partial_{t}\left(\frac{\left|\nabla^{2} u\right|}{u}\right)=\frac{\partial_{t}\left(\left|\nabla^{2} u\right|^{2}\right)}{2 u\left|\nabla^{2} u\right|}-\frac{\left|\nabla^{2} u\right| u_{t}}{u^{2}}
$$

Noting the metric evolves by the Ricci flow, we have

$$
\begin{aligned}
\partial_{t}\left(\left|\nabla^{2} u\right|^{2}\right) & =4 \sum \operatorname{Ric}\left(\nabla u_{j}, \nabla u_{j}\right)+2 \sum u_{i j t} u_{i j}, \\
u_{i j t} & =\partial_{t}\left(u_{i j}-\Gamma_{i j}^{l} u_{l}\right)=u_{t i j}+\sum_{l} u_{l}\left(\nabla_{i} \operatorname{Ric}_{j l}+\nabla_{j} \operatorname{Ric}_{i l}-\nabla_{l} \operatorname{Ric}_{i j}\right) .
\end{aligned}
$$

The Ricci identity gives

$$
u_{i j k k}=u_{k k i j}+\sum_{l}\left(R_{k i k l, j} u_{l}+R_{k i k l} u_{l j}+R_{k j i l} u_{l k}+R_{k j k l} u_{l i}+R_{i j k l, k} u_{l}+R_{i j k l} u_{l k}\right) .
$$

Combining the above and using the Schwarz inequality, we conclude

$$
\left(\Delta-\partial_{t}\right)\left(\frac{\left|\nabla^{2} u\right|}{u}\right) \geq-2 \nabla\left(\frac{\left|\nabla^{2} u\right|}{u}\right) \cdot \nabla \log u-C k_{1} \frac{\left|\nabla^{2} u\right|}{u}-C k_{2} \frac{|\nabla u|}{u} .
$$

Part 2. We calculate the equation for $|\nabla u|^{2} / u^{2}$. A direct computation shows

$$
\begin{aligned}
&(\Delta\left.-\partial_{t}\right)\left(\alpha \frac{|\nabla u|^{2}}{u^{2}}\right) \\
&=2 \alpha \frac{\sum u_{i j}^{2}}{u^{2}}+2 \alpha \frac{\sum u_{i} u_{j j i}}{u^{2}}+2 \alpha \frac{\operatorname{Ric}(\nabla u, \nabla u)}{u^{2}}-8 \alpha \frac{\sum u_{i j} u_{i} u_{j}}{u^{3}}-2 \alpha \frac{\sum u_{i}^{2} u_{j j}}{u^{3}} \\
& \quad+6 \alpha \frac{\sum u_{i}^{2} u_{j}^{2}}{u^{4}}-2 \alpha \frac{\operatorname{Ric}(\nabla u, \nabla u)}{u^{2}}-2 \alpha \frac{\sum u_{i} u_{i t}}{u^{2}}+2 \alpha \frac{\sum u_{i}^{2} u_{t}}{u^{3}} \\
&=2 \alpha \frac{\sum u_{i j}^{2}}{u^{2}}-8 \alpha \frac{\sum u_{i j} u_{i} u_{j}}{u^{3}}+6 \alpha \frac{\sum u_{i}^{2} u_{j}^{2}}{u^{4}} \\
&=-2 \nabla\left(\alpha \frac{|\nabla u|^{2}}{u^{2}}\right) \cdot \nabla \log u+2 \alpha \frac{\sum u_{i j}^{2}}{u^{2}}-4 \alpha \frac{\sum u_{i j} u_{i} u_{j}}{u^{3}}+2 \alpha \frac{\sum u_{i}^{2} u_{j}^{2}}{u^{4}} .
\end{aligned}
$$


In the above, the two Ricci curvature terms generated by the Ricci identity and the evolution of the metric are canceled. Applying Young's inequality, that is,

$$
4 \alpha \frac{\sum u_{i j} u_{i} u_{j}}{u^{3}} \leq 2(1-\delta) \alpha \frac{\sum u_{i j}^{2}}{u^{2}}+\frac{2 \alpha}{1-\delta}\left(\frac{\sum u_{i}^{2}}{u^{2}}\right)^{2} \quad \text { for any } 0<\delta<1,
$$

we conclude

$$
\left(\Delta-\partial_{t}\right)\left(\alpha \frac{|\nabla u|^{2}}{u^{2}}\right) \geq-2 \nabla\left(\alpha \frac{|\nabla u|^{2}}{u^{2}}\right) \cdot \nabla \log u+2 \delta \alpha \frac{\sum u_{i j}^{2}}{u^{2}}-\frac{2 \delta \alpha}{1-\delta}\left(\frac{\sum u_{i}^{2}}{u^{2}}\right)^{2} .
$$

Part 3. Using the formula (2-4) and Young's inequality, we get for any $\varepsilon>0$,

$$
\left(\Delta-\partial_{t}\right)\left(\beta \frac{u_{t}}{u}\right) \geq-2 \nabla\left(\beta \frac{u_{t}}{u}\right) \cdot \nabla \log u-\frac{\beta}{\varepsilon} \frac{\sum u_{i j}^{2}}{u^{2}}-\beta \varepsilon \sum \operatorname{Ric}_{i j}^{2} .
$$

Combining Parts $1-3$ and using (2-6), we obtain for any $0<\delta<1$ and $\varepsilon>0$

$$
\begin{aligned}
\left(\Delta-\partial_{t}\right) F_{1} \geq-2 \nabla F_{1} \cdot \nabla \log u- & C k_{1} \frac{\left|\nabla^{2} u\right|}{u}+\left(\delta \alpha-\frac{\beta}{\varepsilon}\right) \frac{\sum u_{i j}^{2}}{u^{2}}+\delta \alpha \frac{\sum u_{i j}^{2}}{u^{2}} \\
& -\frac{2 \delta \alpha}{1-\delta}\left(\frac{\sum u_{i}^{2}}{u^{2}}\right)^{2}-C k_{2} \frac{|\nabla u|}{u}-4 \beta \varepsilon n^{2} k_{1}^{2} .
\end{aligned}
$$

By the definition of $F_{1}$, we have

$$
\frac{\left|\nabla^{2} u\right|}{u} \leq F_{1}+\beta \frac{u_{t}}{u}
$$

and

$$
\begin{aligned}
\frac{\sum u_{i j}^{2}}{u^{2}} & =\left(F_{1}-\alpha \frac{|\nabla u|^{2}}{u^{2}}+\beta \frac{u_{t}}{u}\right)^{2} \\
& =F_{1}^{2}+\alpha^{2}\left(\frac{\sum u_{i}^{2}}{u^{2}}\right)^{2}+\beta^{2} \frac{u_{t}^{2}}{u^{2}}+2 \beta F_{1} \frac{u_{t}}{u}-2 \alpha F_{1} \frac{\sum u_{i}^{2}}{u^{2}}-2 \alpha \beta \frac{\sum u_{i}^{2}}{u^{2}} \frac{u_{t}}{u} .
\end{aligned}
$$

Inserting (3-5) and (3-6) into (3-4) and applying Young's inequality to separate the mixed items, we arrive at

$$
\begin{aligned}
\left(\Delta-\partial_{t}\right) F_{1} \geq- & 2 \nabla F_{1} \cdot \nabla \log u-C k_{1} F_{1}-\frac{1}{4} C k_{1}^{2} /(\gamma-1)^{2} \\
+ & \left(\frac{1}{2} \delta \alpha \beta^{2}-C \beta^{2}(\gamma-1)^{2}\right) \frac{u_{t}^{2}}{u^{2}}+\left(\delta \alpha-\frac{\beta}{\varepsilon}\right) \frac{\sum u_{i j}^{2}}{u^{2}} \\
- & \left(4 \delta \alpha^{3}+\frac{\delta}{2 \alpha(1-\delta)^{2}}\right)\left(\frac{\sum u_{i}^{2}}{u^{2}}\right)^{2}+\delta \alpha F_{1}^{2} \\
+ & 2 \delta \alpha \beta F_{1} \frac{u_{t}}{u}-2 \delta \alpha^{2} F_{1} \frac{\sum u_{i}^{2}}{u^{2}}-C \delta^{-1 / 3} k_{2}^{4 / 3}-4 \beta \varepsilon n^{2} k_{1}^{2},
\end{aligned}
$$

for any $\gamma-1>0$. 
Using the inequality

$$
\left(\frac{|\nabla u|^{2}}{u^{2}}\right)^{2} \leq 2\left(\frac{|\nabla u|^{2}}{u^{2}}-\gamma \frac{u_{t}}{u}\right)^{2}+2 \gamma^{2} u_{t}^{2} / u^{2}
$$

we calculate

$$
\begin{aligned}
\left(\frac{1}{2} \delta \alpha \beta^{2}-C \beta^{2}(\gamma-1)^{2}\right) \frac{u_{t}^{2}}{u^{2}}- & \left(4 \delta \alpha^{3}+\frac{\delta}{2 \alpha(1-\delta)^{2}}\right)\left(\frac{\sum u_{i}^{2}}{u^{2}}\right)^{2} \\
\geq\left(\frac{1}{2} \delta \alpha \beta^{2}-2 \gamma^{2}\left(4 \delta \alpha^{3}\right.\right. & \left.\left.+\frac{\delta}{2 \alpha(1-\delta)^{2}}\right)-C \beta^{2}(\gamma-1)^{2}\right) \frac{u_{t}^{2}}{u^{2}} \\
& -2\left(4 \delta \alpha^{3}+\frac{\delta}{2 \alpha(1-\delta)^{2}}\right)\left(\frac{|\nabla u|^{2}}{u^{2}}-\gamma \frac{u_{t}}{u}\right)^{2}
\end{aligned}
$$

Setting $\beta=5 \alpha$, we check that

$$
\begin{aligned}
\frac{1}{2} \delta \alpha \beta^{2}-2 \gamma^{2} & \left(4 \delta \alpha^{3}+\frac{\delta}{2 \alpha(1-\delta)^{2}}\right)-C \beta^{2}(\gamma-1)^{2} \\
& =8 \delta \alpha^{3}\left(\frac{25}{16}-\gamma^{2}\right)-\frac{\delta}{\alpha(1-\delta)^{2}} \gamma^{2}-C \beta^{2}(\gamma-1)^{2} \\
& \geq 0 \quad \text { when } \delta>0 \text { and } \gamma-1>0 \text { are sufficiently small. }
\end{aligned}
$$

Then we can take $\varepsilon \geq 5 / \delta$ such that $\delta \alpha-\beta / \varepsilon \geq 0$.

Consequently, (3-7) becomes

$$
\begin{aligned}
\left(\Delta-\partial_{t}\right) F_{1} \geq-2 \nabla & F_{1} \cdot \nabla \log u-C k_{1} F_{1}-\frac{C k_{1}^{2}}{4(\gamma-1)^{2}}+\delta \alpha F_{1}^{2} \\
-2 & \left(4 \delta \alpha^{3}+\frac{\delta}{2 \alpha(1-\delta)^{2}}\right)\left(\frac{|\nabla u|^{2}}{u^{2}}-\gamma \frac{u_{t}}{u}\right) \\
& +2 \delta \alpha \beta F_{1} u_{t} / u-2 \delta \alpha^{2} F_{1} \frac{\sum u_{i}^{2}}{u^{2}}-C k_{2}^{4 / 3}-4 \beta \varepsilon n^{2} k_{1}^{2} .
\end{aligned}
$$

We complete the lemma by applying Corollary 1 and noting that

$$
\left(\Delta-\partial_{t}\right) F=t\left(\Delta-\partial_{t}\right) F_{1}-F_{1} .
$$

Proof of Theorem 4. As in the proof of Theorem 1, $(M, g(t))$ is complete for $t \in[0, T]$. Let

$$
\rho(x, t)=d\left(x, x_{0}, t\right) \quad \text { and } \quad \varphi(x, t)=\psi(\rho(x, t) / R) .
$$

Let $\varphi F(x, t):=\psi(\rho(x, t) / R) F(x, t)$, where $(x, t) \in Q_{2 R, T}$. Suppose $\left(x_{1}, t_{1}\right)$ is the point where $\varphi F$ achieves its maximum in $Q_{2 R, T_{1}}$, where $0<T_{1} \leq T$. 
If $\left|\nabla^{2} u\left(x_{1}, t_{1}\right)\right|=0$, then by Corollary 1 , we have

$$
(\varphi F)\left(x_{1}, t_{1}\right)=\varphi t_{1}\left(\alpha \frac{|\nabla u|^{2}}{u^{2}}-\beta \frac{u_{t}}{u}\right) \leq C_{n, \alpha, \beta}\left(k_{1} t_{1}+1\right),
$$

which implies (3-1).

Using the arguments of [Calabi 1958] and [Li 1991], we can assume that $\varphi F$ is smooth at $\left(x_{1}, t_{1}\right)$ and that $\varphi F\left(x_{1}, t_{1}\right)>0$.

As in the proof of Theorem 1, at the point $\left(x_{1}, t_{1}\right)$, we have

$$
\begin{aligned}
0 & \geq\left(\Delta-\partial_{t}\right)(\varphi F) \\
& \geq\left(-\frac{C}{R^{2}}-\frac{C}{R} \sqrt{k_{1}}\right) F-C k_{1} F+\varphi\left(\Delta_{X}-\partial_{t}\right) F .
\end{aligned}
$$

Applying Lemma 2 and Young's inequality, we obtain for $s>0$ that

$$
\begin{aligned}
& 0 \geq\left(-\frac{C}{R^{2}}-\frac{C}{R} \sqrt{k_{1}}\right) F-C k_{1} F-\frac{F|\nabla \varphi|^{2}}{2 s \varphi}-2 F s \varphi \frac{|\nabla u|^{2}}{u^{2}} \\
&-C k_{1} \varphi F+\frac{\delta \alpha}{t_{1}} \varphi F^{2}-2 C \varphi t_{1}\left(4 \delta \alpha^{3}+\frac{\delta}{2 \alpha(1-\delta)^{2}}\right)\left(1 / t_{1}+k_{1}\right)^{2} \\
&+2 \delta \alpha \beta \varphi F u_{t} / u-2 \delta \alpha^{2} \varphi F \frac{\sum u_{i}^{2}}{u^{2}}-\frac{C k_{1}^{2}}{4(\gamma-1)^{2}} \varphi t_{1} \\
&-C k_{2}^{4 / 3} \varphi t_{1}-4 \beta \varepsilon n^{2} \varphi t_{1} k_{1}^{2}-\varphi F / t_{1} .
\end{aligned}
$$

Using Corollary 1, we have

(3-14) $2 \delta \alpha \beta \varphi F u_{t} / u-2 \delta \alpha^{2} \varphi F \frac{\sum u_{i}^{2}}{u^{2}}-2 F s \varphi \frac{|\nabla u|^{2}}{u^{2}}$

$$
\geq\left(2 \delta \alpha \beta-2 s \gamma-2 \delta \alpha^{2} \gamma\right) \varphi F u_{t} / u-C\left(2 \delta \alpha^{2}+2 s\right) \varphi F\left(1 / t_{1}+K_{1}\right) .
$$

Observe that $2 \delta \alpha \beta-2 s \gamma-2 \delta \alpha^{2} \gamma=0$ when we set $s=\delta \alpha^{2}(4 / \gamma-1)$. Then (3-13) becomes

$$
\begin{gathered}
0 \geq \frac{\delta \alpha}{t_{1}} \varphi F^{2}-\varphi F C\left(2 s+2 \delta \alpha^{2}\right)\left(1 / t_{1}+k_{1}\right)-\frac{C F}{2 s R^{2}}+\left(-\frac{C}{R^{2}}-\frac{C}{R} \sqrt{k_{1}}\right) F \\
-C k_{1} F-C k_{1} \varphi F-\varphi F / t_{1}-\frac{C k_{1}^{2}}{4(\gamma-1)^{2}} \varphi t_{1}-4 \beta \varepsilon n^{2} \varphi t_{1} k_{1}^{2} \\
-C k_{2}^{4 / 3} \varphi t_{1}-2 C \varphi t_{1}\left(4 \delta \alpha^{3}+\frac{\delta}{2 \alpha(1-\delta)^{2}}\right)\left(1 / t_{1}+k_{1}\right)^{2}
\end{gathered}
$$


Multiplying through by $\varphi t_{1}$ and using $0 \leq \varphi \leq 1$, we have

$$
\begin{aligned}
0 \geq & \delta \alpha(\varphi F)^{2}-(\varphi F)\left(\left(\frac{C}{R^{2}}+\frac{C}{R} \sqrt{k_{1}}\right) t_{1}+\left(8 C \delta \alpha^{2} / \gamma\right)\left(1 / t_{1}+k_{1}\right) t_{1}\right) \\
& -(\varphi F)\left(\frac{C t_{1}}{2 \delta \alpha^{2}(4 / \gamma-1) R^{2}}+\left(1+C k_{1} t_{1}\right)\right)-\frac{C k_{1}^{2}}{4(\gamma-1)^{2}} t_{1}^{2} \\
& -2 C\left(4 \delta \alpha^{3}+\frac{\delta}{2 \alpha(1-\delta)^{2}}\right)\left(1 / t_{1}+k_{1}\right)^{2} t_{1}^{2}-4 \beta \varepsilon n^{2} k_{1} t_{1}^{2}-C k_{2}^{4 / 3} t_{1}^{2} .
\end{aligned}
$$

Applying the quadratic formula, we get

$$
\varphi F\left(x_{1}, t_{1}\right) \leq C\left(1+k_{1} t_{1}+k_{2}^{2 / 3} t_{1}+\left(C / R^{2}\right) t_{1}\right) .
$$

By an argument similar to one in the proof of Theorem 1, we conclude

$$
F_{1}(x, t) \leq C\left(k_{1}+k_{2}^{2 / 3}+1 / t+1 / R^{2}\right) \quad \text { in } Q_{R, T},
$$

where $C$ depends on $n$ and $\alpha$. Because $M$ is noncompact, we can let $R \rightarrow+\infty$. This completes the proof of Theorem 4 .

\section{Acknowledgment}

We thank Professor Jiayu Li for his guidance and encouragement. We also thank Yunyan Yang and Jun Sun for their very useful suggestions.

\section{References}

[Calabi 1958] E. Calabi, "An extension of E. Hopf's maximum principle with an application to Riemannian geometry”, Duke Math. J. 25 (1958), 45-56. MR 19,1056e Zbl 0079.11801

[Chow and Knopf 2004] B. Chow and D. Knopf, The Ricci flow: An introduction, Mathematical Surveys and Monographs 110, American Mathematical Society, Providence, RI, 2004. MR 2005e: $53101 \mathrm{Zbl} 1086.53085$

[Chow et al. 2006] B. Chow, P. Lu, and L. Ni, Hamilton's Ricci flow, Graduate Studies in Mathematics 77, American Mathematical Society, Providence, RI, 2006. MR 2008a:53068 Zbl 1118.53001

[Guenther 2002] C. M. Guenther, "The fundamental solution on manifolds with time-dependent metrics”, J. Geom. Anal. 12:3 (2002), 425-436. MR 2003a:58034 Zbl 1029.58018

[Hamilton 1982] R. S. Hamilton, "Three-manifolds with positive Ricci curvature", J. Differential Geom. 17:2 (1982), 255-306. MR 84a:53050 Zbl 0504.53034

[Hamilton 1995] R. S. Hamilton, "The formation of singularities in the Ricci flow", pp. 7-136 in Surveys in differential geometry, II (Cambridge, MA, 1993), edited by S.-T. Yau, International, Cambridge, MA, 1995. MR 97e:53075 Zbl 0867.53030

[Hamilton 1997] R. S. Hamilton, "Four-manifolds with positive isotropic curvature", Comm. Anal. Geom. 5:1 (1997), 1-92. MR 99e:53049 Zbl 0892.53018

[Hamilton 1999] R. S. Hamilton, "Non-singular solutions of the Ricci flow on three-manifolds", Comm. Anal. Geom. 7:4 (1999), 695-729. MR 2000g:53034 Zbl 0939.53024 
[Kuang and Zhang 2008] S. Kuang and Q. S. Zhang, "A gradient estimate for all positive solutions of the conjugate heat equation under Ricci flow", J. Funct. Anal. 255:4 (2008), 1008-1023. MR 2433960 Zbl 1146.58017

[Li 1991] J. Y. Li, "Gradient estimate for the heat kernel of a complete Riemannian manifold and its applications", J. Funct. Anal. 97:2 (1991), 293-310. MR 92f:58174 Zbl 0724.58064

[Li 1994] J. Y. Li, "The Sobolev inequality and Sobolev imbedding theorem for Riemannian manifolds with nonnegative Ricci curvature", Chinese Ann. Math. Ser. A 15:4 (1994), 461-471. In Chinese. MR 96e:58146

[Li and Yau 1986] P. Li and S.-T. Yau, "On the parabolic kernel of the Schrödinger operator", Acta Math. 156:3-4 (1986), 153-201. MR 87f:58156

[Perelman 2002] G. Perelman, "The entropy formula for the Ricci flow and its geometric applications", preprint, 2002. arXiv math.DG/0211159

[Perelman 2003] G. Perelman, "Ricci flow with surgery on three manifolds", preprint, 2003. arXiv math.DG/0303109

[Shi 1989] W.-X. Shi, "Deforming the metric on complete Riemannian manifolds", J. Differential Geom. 30:1 (1989), 223-301. MR 90i:58202 Zbl 0676.53044

[Zhang 2006] Q. S. Zhang, "Some gradient estimates for the heat equation on domains and for an equation by Perelman”, Int. Math. Res. Not. 2006, Art. ID 92314 (2006). MR 2007f:35116

[Zhang 2007] Q. S. Zhang, "A uniform Sobolev inequality under Ricci flow", Int. Math. Res. Not. IMRN 2007, Art. ID rnm056 (2007). Corrected in 2007, Art. ID rnm096 (2007). MR 2008g:53083

Received September 10, 2008. Revised September 28, 2008.

\section{SHIPING LIU}

ACADEMy of Mathematics And Systems SCIENCE

CHINESE ACADEMY OF SCIENCES

BEIJING 100190

CHINA

liushiping@amss.ac.cn 